\title{
3D Guide Wire Reconstruction from Biplane Image Sequences for 3D Navigation in Endovascular Interventions
}

\author{
S.A.M. Baert, E.B. van der Kraats, and W.J. Niessen \\ Image Sciences Institute, University Medical Center Utrecht \\ Rm E 01.334, P.O.Box 85500, 3508 GA Utrecht, The Netherlands \\ \{shirley, everine,wiro\}@isi.uu.nl
}

\begin{abstract}
Using 3D rotational X-ray angiography (3DRA), 3D information of the vasculature can be obtained prior to endovascular interventions. However, during interventions, the radiologist has to rely on fluoroscopy images to manipulate the guide wire. In order to take full advantage of the 3D information from 3DRA data during endovascular interventions, a method is presented which yields an integrated display of the position of the guide wire and vasculature in $3 \mathrm{D}$. The method relies on an automated method developed by the authors that simultaneously tracks the guide wire in biplane fluoroscopy images. Based on the known geometry, the $3 \mathrm{D}$ guide wire position is reconstructed and visualized in the $3 \mathrm{D}$ coordinate system of the vasculature. The method is illustrated in an intracranial anthropomorphic vascular phantom.
\end{abstract}

\section{Introduction}

In response to the demand for minimally invasive interventions, the number of endovascular interventions has increased rapidly over the last years. During these interventions, a guide wire is inserted into the groin and advanced under fluoroscopic guidance. Due to the complexity of the vasculature, and the narowness of the blood vessels, accurate positioning of the guide wire is difficult, especially during neuro-interventions. This results in prolonged examination times and hence prolonged exposure to X-rays for patients and medical staff.

The recent introduction of motorized calibrated X-ray angiography systems allows $3 \mathrm{D}$ reconstruction of the vasculature by performing a 180 degree rotation around the patient. During a rotation a large number of images is taken from known positions. For all positions the geometric distortions have been estimated in a calibration procedure and hence they can be corrected for. Whereas the 3D reconstruction can be used before starting endovascular interventions, the acquisition time is too long to acquire images during an intervention. In this paper a method is developed which, based on the position of the guide wire in calibrated biplane fluoroscopic projection images, reconstructs the 3D guide wire position and subsequently projects this position on the $3 \mathrm{D}$ volume reconstruction of the vasculature. This indicates how the guide wire is positioned with respect to the vasculature and can be used as a $3 \mathrm{D}$ navigation tool for radiologists. 
Whereas a number of researchers have used biplane acquisitions in order to reconstruct the centerlines of the vascular tree 589, or to determine other objects in 3D [4], dynamically relating the guide wire position to prior obtained $3 \mathrm{D}$ vasculature is new.

This paper is organized as follows: In Section 2 the 2D tracking procedure is presented, which is based on the energy minimization of a spline parameterization of the guide wire in a feature image where line-like structures are enhanced. In Section 3 the calibration procedure to correct for distortion and to determine the geometrical projection parameters of the biplane fluoroscopic images is discussed. Section 4 presents the determination of the correspondence of the splines in the biplane images and the reconstruction of the guide wire in $3 \mathrm{D}$ based on the knowledge of the projection geometry. In Section [5] the results are presented and Section [6] concludes with a discussion.

\section{Guide Wire Tracking in 2D}

The 2D guide wire tracking procedure used in this paper is an extension to a method previously presented [1]. The main steps of the algorithm are briefly summarized here. The guide wire is simultaneously tracked in images acquired using a biplane system. In order to represent the guide wire, a third order spline parameterization is used. In the tracking method, first the spline is positioned on the guide wire and subsequently the spline is moved towards the tip of the guide wire for more accurate tip localization.

In order to find the spline in frame $n+1$ if the position in frame $n$ is known, first a rough displacement of the guide wire is estimated. Hereto a template is constructed which is rigidly registered to a feature image using cross-correlation. In the feature image line-like structures are enhanced by analyzing the eigenvalues of the Hessian matrix, which is computed with scaled Gaussian derivative operators. The largest absolute eigenvalue of the Hessian matrix has a high output on line-like structures. The sign of this eigenvalue determines whether it concerns a dark (positive) or a bright (negative) structure. Since a guide wire is a dark elongated structure on a brighter background, the feature image is set to the largest eigenvalue if positive and to zero otherwise.

Subsequently, the spline is fitted on the feature image using an energy minimization approach (Powell's direction set method), using internal constraints which are related to the geometry of the curve (curvedness) and external constraints which depend on greyvalue and directional information contained in the feature image. In this step the inner product between the spline and the orientation of the feature is used. This assures that the spline achieves a similar orientation as the guide wire in the image.

After this step, the endpoint is not necessarily positioned on the endpoint of the guide wire, especially in case of guide wires with a straight tip. In order to the determine the endpoint, the length of the guide wire is increased at the tip, while fixing the tail position. Tip localization is then achieved using a discriminant 
function based on the gradient of the feature image along the spline and the distance to the endpoint position of the guide wire in the previous frame.

\subsection{Acquisition}

For the experiments an intracranial anthropomorphic vascular phantom which is filled with contrast material (Ultravist-300 (Schering, Weesp, the Netherlands), diluted to $50 \%$ with Natriumchloride $0.9 \%$ (Fresenius, 's-Hertogenbosch, the Netherlands)) is used. The rotational angiography facility of a Philips Integris BV5000 C-arm imaging system was used to acquire $100 \mathrm{X}$-ray images at different views by automatic rotation of the C-arm over 180 degrees in about eight seconds. All projection images have a matrix size of $512 \times 512$ pixels. Finally a filtered back-projection algorithm [3], which is a modification of Feldkamp's cone-beam algorithm [2] was applied to generate a 3D reconstruction with a resolution of $128 \times 128 \times 128$ voxels. A biplane image sequence of 25 images was obtained, while advancing the guide wire in the phantom.

\section{Calibration}

In order to relate the coordinates in the $2 \mathrm{D}$ projection images to world coordinates, the acquired biplane projection data have to be corrected for various types of distortion. Pincushion distortion (curved input screen) and the earth magnetic field variations (s-shaped distortion) which are different for each projection direction have to be corrected for, in order to guarantee correlation of corresponding pixels (see [6[7]). These distortions are measured using a Cartesian-grid phantom of which a $3 \mathrm{D}$ acquisition is made. For all projection angles, the distortion is modelled using bivariate polynomials. The distortion correction is performed with subpixel accuracy. Additionally the focal spot position is determined. Furthermore, the projection geometry is measured, since the mechanical bending of the $\mathrm{C}$-arm causes the isocentre not being constant during the rotation.

\section{3D Reconstruction from Biplane Images}

In order to reconstruct the guide wire in 3D world coordinates, pairs of corresponding points have to be determined in the two splines that have been calculated using 2D tracking in the biplane images. Since the projection parameters and the locations and orientations of the focal spot are known, the correspondence can be solved using the epipolar constraint.

Figure 1 illustrates the three independent coordinate systems: the volume system $\left\{\boldsymbol{v}_{x}, \boldsymbol{v}_{y}, \boldsymbol{v}_{z}\right\}$, the detector system $\left\{\boldsymbol{d}_{x}, \boldsymbol{d}_{y}, \boldsymbol{d}_{z}\right\}$ which moves relative to the other systems due to the rotation of the image intensifier, and a global system $\left\{\boldsymbol{g}_{x}, \boldsymbol{g}_{y}, \boldsymbol{g}_{z}\right\}$, represented by the global origin $O$.

Given a point on the spline in the first image, we are now looking for the corresponding point on the spline in the other image. Therefore first the epipolar 


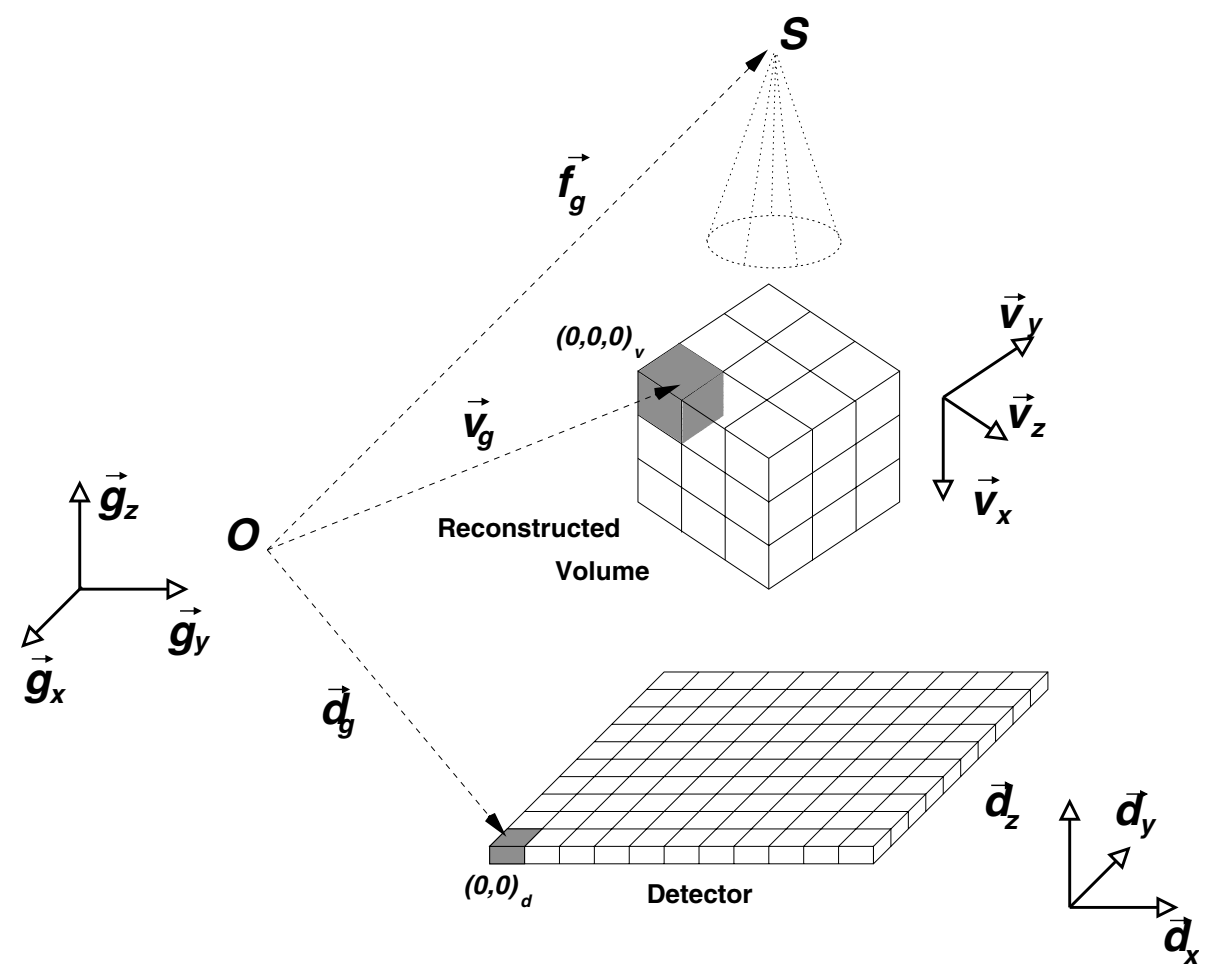

Fig. 1. Illustration of the geometric framework, in which three independent coordinate systems coexist: the volume system $\mathbf{v}$, the detector system $\mathbf{d}$, and a global system $\mathbf{g}$. $S$ is the position of the $X$-ray source.

line is calculated. Given a point $P_{a}$ on the spline representing the guide wire in image $A$ :

$$
\boldsymbol{P}_{a}=\left(\begin{array}{l}
x \\
y
\end{array}\right)
$$

then the transformation to global coordinates is given by

$$
\boldsymbol{P}_{a, g l o b a l}=\boldsymbol{d}_{a, g l o b a l}+x p_{d x} \cdot \boldsymbol{d}_{a, x}+y p_{d y} \cdot \boldsymbol{d}_{a, y}
$$

where $\boldsymbol{d}_{a, g l o b a l}$ is the vector pointing from the global origin to the lower left corner of the detector plane (see Figure 1) and $\boldsymbol{d}_{a, x}$ and $\boldsymbol{d}_{a, y}$ are the detector normals of image $A$ in the x- and y-direction. The epipolar line is obtained by the intersection of plane $H$, which is defined by the point $\boldsymbol{P}_{a, g l o b a l}$ and the two focal spots $\boldsymbol{f}_{a}$ and $\boldsymbol{f}_{b}$, and the image plane $B$, see Figure 2 .

The corresponding point $\boldsymbol{P}_{b}$ in image $B$ can be generated by calculating the intersection of the epipolar line and the spline, representing the guide wire, in image $B$. If there is more than one intersection, the point of the intersection nearest to the previous intersection point is taken as the corresponding point. Intersection of the line defined by $\boldsymbol{P}_{a, g l o b a l}$ and $\boldsymbol{f}_{a}$, and the line defined by 


\section{$\begin{array}{ll}\text { Plane A } & \text { Plane B }\end{array}$}

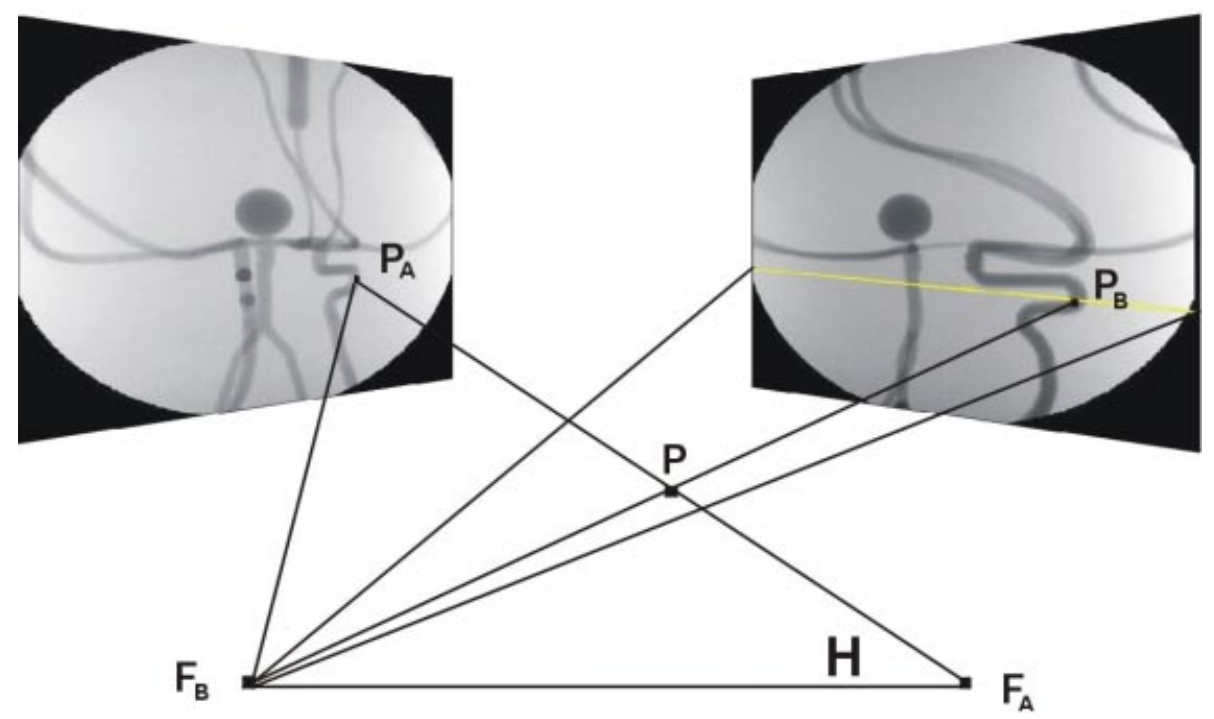

Fig. 2. Schematic example of the epipolar concept.

$\boldsymbol{P}_{b, \text { global }}$ and $\boldsymbol{f}_{b}$, gives point $\boldsymbol{P}$ in $3 \mathrm{D}$. This approach is iteratively applied for all points in the spline from tip to tail, so as to construct full correspondence of the splines in the fluorosopic images and the guide wire position in $3 \mathrm{D}$ world coordinates.

\section{Results}

In this paper experiments were carried out, involving a phantom for which a $3 \mathrm{D}$ reconstruction of the vasculature and biplane image sequences in which a guide wire is advanced, are available. Independent $2 \mathrm{D}$ guide wire tracking in each of the two projection planes is applied using a method previously presented [1. In this method the guide wire could accurately be tracked in $96 \%$ of the frames. Subsequently corresponding points are determined using the epipolar constraint. $3 \mathrm{D}$ reconstruction is then performed and the $3 \mathrm{D}$ position of the guide wire is obtained in the same coordinate system as the $3 \mathrm{D}$ reconstruction of the vasculature. Therefore, the position of the guide wire in the vasculature can be visualized. Figure 3 shows the calculated position of the guide wire projected into the 3DRA data for four timepoints in the image sequence. It can be observed that the estimated position is within the vasculature.

\section{Discussion}

A method has been presented which, based on simultaneously tracking a guide wire in biplane fluoroscopic images, reconstructs the guide wire position in $3 \mathrm{D}$ 

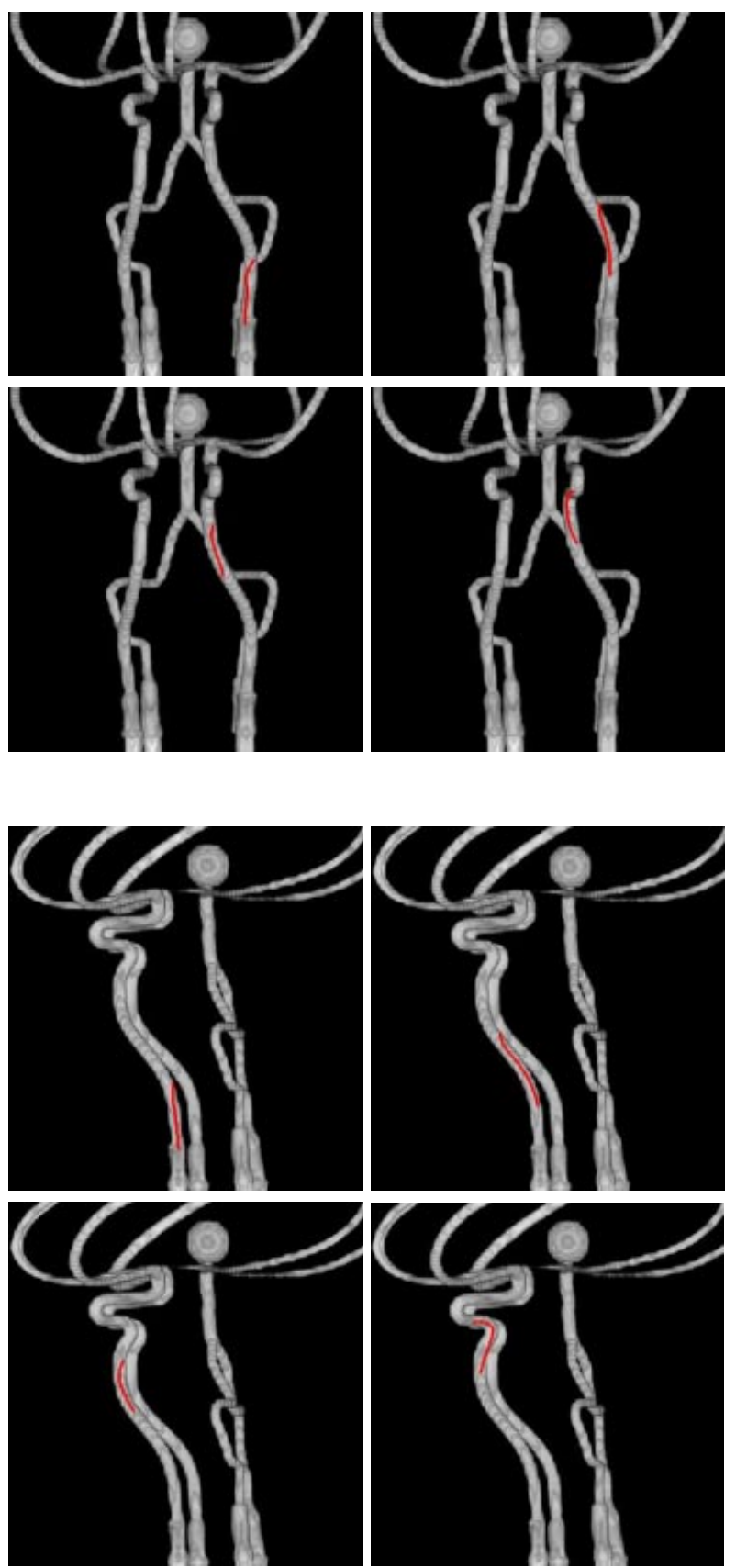

Fig. 3. Four non-subsequent frames, 1, 10, 15 and 20 of the image sequences with the frontal images (upper four) and lateral images (lower four). It can be observed that the estimated guide wire position is inside the vasculature at all timepoints. For color images, see the digital version of this paper. 
during endovascular interventions. Since this position is expressed in the same coordinate system as in which the pre-intervention 3D reconstructions of the vasculature have been made, this approach enables a $3 \mathrm{D}$ visualization of the guide wire in the vasculature which provides a 3D navigational tool for interventional radiologists. In order to improve the accuracy of $3 \mathrm{D}$ guide wire position estimation, the 2D biplane images have been corrected for pincushion distortion, distortions due to the earth magnetic field, and the projection geometry has been measured to compensate for mechanical bending of the C-arm. In an anthropomorphic phantom it was shown that the estimated position of the guide wire coincides with the position of the vasculature in the pre-intervention images. In order to validate the estimated guide wire position, and to assess the influence of correcting for the distortions, a series of experiments will be initiated in which $3 \mathrm{D}$ reconstructions of the guide wire will be made next to $2 \mathrm{D}$ fluoroscopic sequences, so as to provide a gold standard for the 3D guide wire position.

\section{References}

1. S.A.M. Baert, W.J. Niessen, A.F. Frangi, E.H.W. Meijering and M.A. Viergever, Guide Wire Tracking during Endovascular Interventions, Proceedings of MICCAI 2000, Lecture Notes in Computer Science Vol. 1935, pp. 727-734.

2. L.A. Feldkamp, L.C. Davis, J.W. Kress, Practical Cone-Beam Algorithm, Journal of the Optical Society of America A. 1984, Vol. 1, No. 6, pp. 612-619.

3. M. Grass, R. Koppe, E. Klotz et al., 3D Reconstruction of High Contrast Objects using C-Arm Image Intensifier Projection Data, Computerized Medical Imaging and Graphics 1999, Vol. 23 pp. 311-321.

4. K.R. Hoffmann, B.B. Williams, J. Esthappan et al., Determination of $3 D$ Positions of Pacemaker Leads from Biplane Angiographic Sequences, Medical Physics 1997, Vol. 24, No. 12, pp. 1854-1862.

5. K.R. Hoffmann, A. Sen, L. Lan et al., A System for Determination of 3D Vessel Tree Centerlines form Biplane Images, International Journal of Cardiac Imaging 2000, Vol.16, No.5: pp. 315-330.

6. R. Koppe, E. Klotz, J. Op de Beek and H. Aerts, 3D Vessel Reconstruction based on Rotational Angiography, Proceedings of CAR 1995, pp. 103.

7. R. Koppe, E. Klotz, J. Op de Beek and H. Aerts, Digital stereotaxy/stereotactic procedures with C-arm based Rotational Angiography, Proceedings of CAR 1996, pp. $17-22$.

8. B. Movassaghi 3D Reconstruction by Modeling of Coronary Arteriograms, Diploma Thesis, Heinrich-Heine University, Düsseldorf, June 2001.

9. L. Sarry, J.Y. Boire, Three-dimensional Tracking of Coronary Arteries from Biplane Angiographic Sequences using Parametrically Deformable Models, IEEE Transactions on Medical Imaging 2001, Vol.20, No. 12, pp. 1341-1351. 